\title{
Inclusion body formation reduces levels of mutant huntingtin and the risk of neuronal death
}

\author{
Montserrat Arrasate ${ }^{1,2}$, Siddhartha Mitra ${ }^{1,3,4}$, Erik S. Schweitzer ${ }^{5}$, Mark R. Segal ${ }^{6}$ \& Steven Finkbeiner ${ }^{1,2,3,4,7}$ \\ ${ }^{1}$ Gladstone Institute of Neurological Disease, ${ }^{2}$ Neuroscience Program, ${ }^{3}$ Biomedical Sciences Program, and ${ }^{4}$ Medical Scientist Training Program, University of California, \\ San Francisco, California 94141, USA \\ ${ }^{5}$ Brain Research Institute, University of California School of Medicine, Los Angeles, California 90095-1761, USA \\ ${ }^{6}$ Division of Biostatistics, University of California, San Francisco, California 94143-0560, USA \\ ${ }^{7}$ Departments of Neurology and Physiology, University of California, San Francisco, California 94141, USA
}

Huntington's disease is caused by an abnormal polyglutamine expansion within the protein huntingtin and is characterized by microscopic inclusion bodies of aggregated huntingtin and by the death of selected types of neuron. Whether inclusion bodies are pathogenic, incidental or a beneficial coping response is controversial. To resolve this issue we have developed an automated microscope that returns to precisely the same neuron after arbitrary intervals, even after cells have been removed from the microscope stage. Here we show, by survival analysis, that neurons die in a time-independent fashion but one that is dependent on mutant huntingtin dose and polyglutamine expansion; many neurons die without forming an inclusion body. Rather, the amount of diffuse intracellular huntingtin predicts whether and when inclusion body formation or death will occur. Surprisingly, inclusion body formation predicts improved survival and leads to decreased levels of mutant huntingtin elsewhere in a neuron. Thus, inclusion body formation can function as a coping response to toxic mutant huntingtin.

Huntington's disease (HD), a neurodegenerative disorder caused by an abnormal polyglutamine (polyQ) expansion within the protein huntingtin (Htt), is characterized by the aggregation of Htt into microscopic intracellular deposits called inclusion bodies (IBs) and by the death of striatal and cortical neurons. However, the relationship between Htt deposition and neurodegeneration is controversial. Sometimes IB formation has been associated with neurodegeneration ${ }^{1-4}$; at other times, there was no or a negative correlation $^{5-9}$. Three competing models have described IB formation as pathogenic, incidental or a beneficial coping response $e^{5,10-12}$.

IBs seem to result from aggregation that generates many protein complexes differing in multimerization and three-dimensional structure $^{13}$. These complexes often coexist with IBs, but low temporal and spatial resolution have limited the interpretation of past experiments that correlated IB formation with neurodegeneration. Attempts to disrupt the aggregation process yielded opposing results, depending on the manipulation ${ }^{5,7,9,14-16}$, probably because it is impossible to manipulate aggregation selectively ${ }^{17}$.

\section{Automated microscopy of a model for HD}

To increase the temporal resolution of conventional approaches, we developed an automated microscope system ${ }^{18}$ that returns to precisely the same neuron or field of neurons, even after cells have been removed from the microscope stage during the interval. We prospectively measured the survival of individual neurons, the intracellular levels of mutant Htt and the aggregation of Htt into IBs. The relationships between these factors were determined by survival analysis without introducing potentially confounding nonspecific manipulations ${ }^{19,20}$.

We examined an established neuronal HD model ${ }^{5}$ in which striatal neurons are transiently transfected with Htt. The model recapitulates several $\mathrm{HD}$ features (for example, IB formation and polyQ-expansion-dependent, neuron-specific death $)^{5}$. To reveal Htt in living striatal neurons, we used amino-terminal exon 1 fragments of $\mathrm{Htt}\left(\mathrm{Htt}^{\mathrm{ex} 1}\right)$ containing polyQ stretches of various lengths and fused to the $\mathrm{N}$ terminus of green fluorescent protein $(\mathrm{GFP})^{21}$. A similar fragment may be generated in HD by proteolytic cleavage $e^{22-26}$ and is sufficient to produce HD-like features when expressed as a transgene in a mouse $\mathrm{e}^{27}$. Along with $\mathrm{Htt}^{\mathrm{ex} 1}-\mathrm{GFP}$, neurons were cotransfected with a monomeric red fluorescent protein $(\mathrm{mRFP})^{28}$ to reveal neurons independently of $\mathrm{Htt}^{\mathrm{ex} 1}$-GFP (Supplementary Fig. S1). Fluorescent protein expression and periodic imaging did not affect neuronal viability ${ }^{18}$.

Neurons were imaged with the automated microscope 2-24 h after transfection and at 12-24-h intervals (Fig. 1a). Some neurons abruptly lost mRFP fluorescence. This event corresponded to the loss of membrane integrity and cell death and was well correlated with other cell-death markers (Fig. 1b, Supplementary Fig. S2). Others have found the loss of a fluorescent marker protein to be a highly sensitive and specific assay of cell death through different pathways and in different types of cell ${ }^{29}$. The ability to monitor individual neurons over time allows us to quantify differences in their longevity by survival analysis ${ }^{19,20}$. We determined the survival function for neurons transfected with GFP or with $\mathrm{Htt}^{\mathrm{ex} 1}$-GFP containing a normal $\left(\mathrm{Q}_{17}\right)$ or expanded $\left(\mathrm{Q}_{72}\right)$ polyQ stretch. Neurons transfected with $\mathrm{Htt}^{\mathrm{ex} 1}$-GFP containing disease-associated polyQ stretches died faster than neurons transfected with $\mathrm{Htt}^{\mathrm{ex} 1}-\mathrm{Q}_{17}-\mathrm{GFP}$ (Fig. 1c).

From the survival functions, we deduced hazard functionsthe estimated instantaneous risk of death of individual cells, independent of population size ${ }^{19,20}$. The cumulative risk of death was similar and remained relatively low in neurons transfected with GFP or $\mathrm{Htt}^{\mathrm{ex1}}-\mathrm{Q}_{17}-\mathrm{GFP}$ (Fig. 1d, Supplementary Fig. S3). However, $\mathrm{Htt}^{\mathrm{ex} 1}-\mathrm{Q}_{47}-\mathrm{GFP}, \mathrm{Htt}^{\mathrm{ex} 1}-\mathrm{Q}_{72}$-GFP or $\mathrm{Htt}^{\mathrm{ex} 1}-\mathrm{Q}_{103}$-GFP significantly increased the risk, and the increase was correlated with the length of the polyQ stretch. These results parallel features of HD: polyQ stretches longer than $35 \mathrm{Q}$ can cause neurodegeneration, with symptoms appearing sooner for longer stretches ${ }^{30}$.

Knowing whether the risk of death changes over time can provide insights into the mechanisms responsible for neurodegeneration ${ }^{31}$. The cumulative risk of death increases as cells continually die 
(Fig. 1), but the risk of cell death does not necessarily change. To determine whether the risk of death changes, we tested the linearity of the non-cumulative hazard function: a curved function means that the risk of death changes over time; linearity indicates that the risk is largely time-independent. The hazard functions for neurons transfected with $\mathrm{Htt}^{\mathrm{ex} 1}-\mathrm{Q}_{47}-\mathrm{GFP}, \mathrm{Htt}^{\mathrm{ex} 1}-\mathrm{Q}_{72}$-GFP or $\mathrm{Htt}^{\mathrm{ex} 1}-\mathrm{Q}_{103^{-}}$ GFP were essentially linear ( $F$-test, not significant), indicating that the expanded polyQ stretches increase the risk of death relatively constantly over time.

However, these cultures contain subtypes of striatal neuron whose susceptibility varies in $\mathrm{HD}^{5,32,33}$ and could mask a temporal variation in the risk of death conferred by polyQ expansion. We therefore performed parallel experiments in a homogeneous, neuronlike phaeochromocytoma 12 (PC12) cell line (Fig. 1e, f). PC12 cells containing versions of $\mathrm{Htt}$ with disease-associated polyQ expansions had a higher risk of death than those containing versions of Htt with wild-type polyQ expansions; the increase was relatively constant over time, as in primary striatal neurons. We conclude that polyQ expansion beyond the disease threshold length leads to a steady but increased risk of cell death. These findings offer the first direct test and support of a recently proposed model of HD neurodegeneration inferred from pathological specimens ${ }^{31}$.

To examine IB formation and neuronal death, we first sought to confirm that we could detect and monitor IBs in live neurons. We previously reported that in cultured striatal neurons, polyQexpanded Htt forms IBs that label with antibodies against ubiqui$\operatorname{tin}^{5}$, as in HD. As with other cell types ${ }^{34,35}$, some neurons containing polyQ-expanded Htt fused to GFP developed punctate, highly fluorescent intracellular structures resembling IBs (Fig. 1a, white and yellow arrows in bottom row; Fig. 2a). To characterize these structures further, we fixed GFP-tagged $\mathrm{Htt}$ in situ and measured its fluorescence before and after treatment with detergent ${ }^{21}$. GFP fluorescence in the structures was not significantly affected, but was almost completely destroyed elsewhere in the neuron, indicating that these structures were possibly IBs (Fig. 2a, b).

Because the fluorescence intensity of $\mathrm{Htt}^{\mathrm{ex} 1}$-GFP within IBs is almost fivefold that of diffuse $\mathrm{Htt}^{\mathrm{exl}}$-GFP elsewhere in the neuron, we used this distinction to identify IBs within living neurons and to follow their fates longitudinally. IBs formed in neurons transfected with $\mathrm{Htt}^{\mathrm{ex} 1}-\mathrm{Q}_{47}-\mathrm{GFP}, \mathrm{Htt}^{\mathrm{ex} 1}-\mathrm{Q}_{72}-\mathrm{GFP}$ or $\mathrm{Htt}^{\mathrm{ex} 1}-\mathrm{Q}_{103}-\mathrm{GFP}$ but not with $\mathrm{Htt}^{\mathrm{ex} 1}-\mathrm{Q}_{17}-\mathrm{GFP}$ or $\mathrm{Htt}^{\mathrm{exl}}-\mathrm{Q}_{25}-\mathrm{GFP}$. IBs became detectable at less than $1 \mu \mathrm{m}^{2}$ and achieved sizes similar to those in $\mathrm{HD}^{2,3,36}$, typically growing for as long as the neuron remained alive (Fig. 2c). Larger IBs are also more common in later stages of HD (ref. 36). Thus, the size and behaviour of IBs formed by Htt in transfected striatal neurons resemble those seen in HD.

\section{Death without IB formation}

If IBs trigger neuronal death through gradual sequestration and functional loss of other critical cellular proteins ${ }^{37}$, functional loss of these critical proteins-and therefore the risk of death-should increase with the number and size of IBs (that is, the IB load). Over time, the size of IBs (Fig. 2c) and the fraction of neurons that contain them (that is, the prevalence; Fig. 2d) increased significantly. However, the risk of death from polyQ expansion is relatively constant (see above), indicating that IB load is unlikely to explain polyQ-dependent cell death.

Could an earlier form of polyQ-expanded Htt be the principal toxic species? To test this possibility we recorded the moment at which an IB was first detected (that is, the IB incidence) and measured its relationship to polyQ-dependent death. It has not been possible to measure IB incidence before because conventional approaches fail to record neurons that form IBs but die before they are detected and scored. IB incidence for $\mathrm{Htt}^{\mathrm{ex} 1}-\mathrm{Q}_{103}-\mathrm{GFP}$ was more a
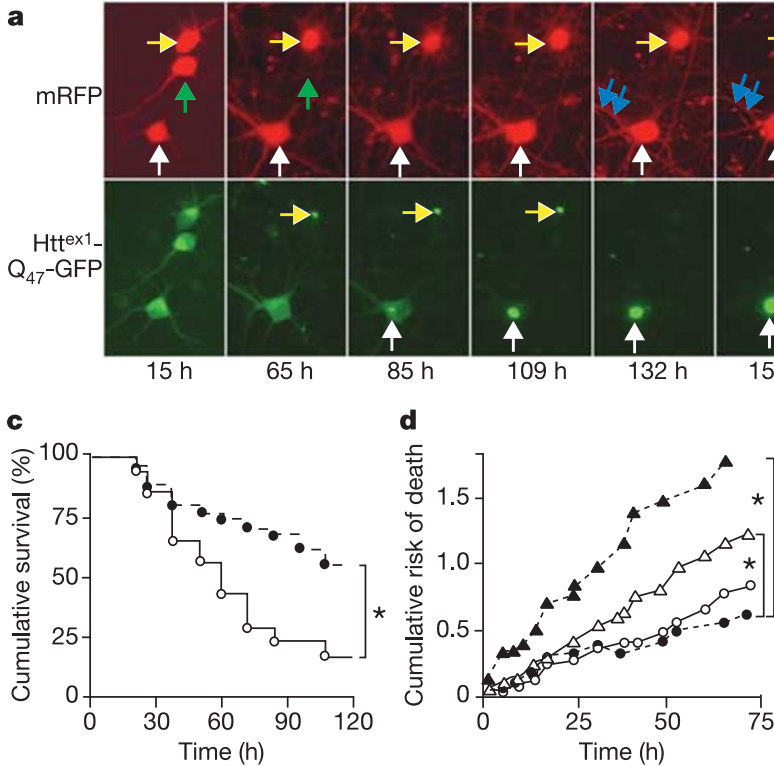

Figure 1 PolyQ-expansion-dependent cell death measured with an automated microscope. a, Longitudinal tracking of single neurons expressing mRFP (top panels) and $\mathrm{Htt}^{\mathrm{ex}}-\mathrm{Q}_{47}$-GFP (bottom panels). Two neurons (yellow and white arrows, top row) that formed IBs (yellow and white arrows, bottom row) outlived a third neuron, which died without an IB (green arrow). Soon after an IB formed (white arrow, bottom panel), mutant $\mathrm{Htt}$ disappeared elsewhere in the neuron. Neuron morphology remained intact for days (top row), but then neurites degenerated (blue arrows) and the neuron died. b, Abrupt loss of marker protein fluorescence (white arrows, compare two bottom left panels) is accompanied by staining with the nuclear dye ethidium bromide (EtBr), indicating death (white arrows; compare two bottom right panels). c, Survival analysis of neurons transfected with wild-type ( $\mathrm{Htt}^{\mathrm{ex} 1}-\mathrm{Q}_{17}-\mathrm{GFP}$, filled circles) or mutant $\left(\mathrm{Htt}{ }^{\mathrm{ex} 1}-\mathrm{Q}_{72}-\mathrm{GFP}\right.$, open
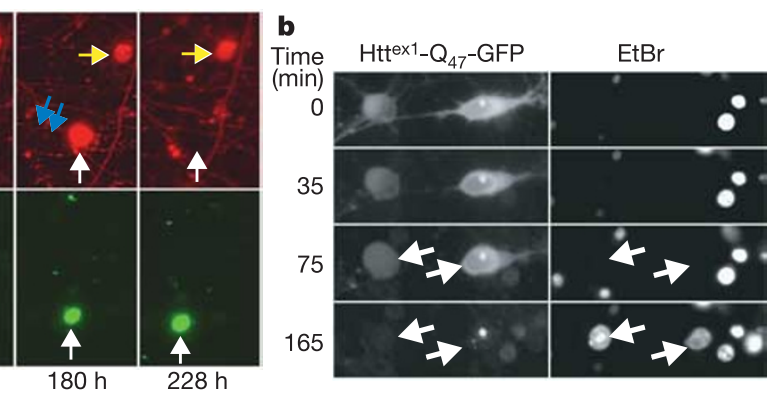

e
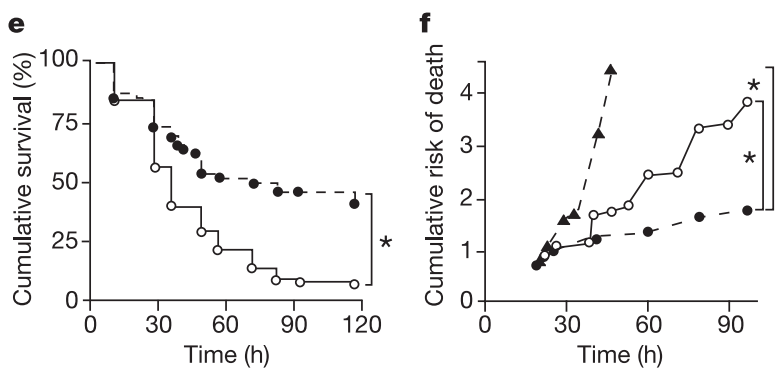

circles) Htt illustrates polyQ-expansion-dependent death ( $n>100$ neurons, four experiments).

d, Hazard analysis demonstrates that versions of $\mathrm{Htt}$ with disease-associated polyQ expansions increase the risk of death significantly and in a length-dependent fashion $(n=4)$. Filled triangles, $\mathrm{Htt}^{\mathrm{ex} 1}-\mathrm{Q}_{103}$-GFP; open triangles, $\mathrm{Htt}^{\mathrm{ex} 1}-\mathrm{Q}_{72}$-GFP; filled circles, $\mathrm{Htt}^{\mathrm{ex} 1}-\mathrm{Q}_{17}$-GFP; open circles, GFP. e, f, Homogeneous PC12 cells that are either stably (e) or transiently (f) transfected with Htt-GFP undergo a polyQ-expansion-dependent decrease in survival and corresponding increase in death risk ( $n>200$ PC12 cells, two or three experiments). Symbols in e: filled circles, $\mathrm{Htt}^{\mathrm{ex} 1}-\mathrm{Q}_{25}$-GFP; open circles, $\mathrm{Htt}^{\mathrm{ex}}{ }_{\text {- }}$ $\mathrm{Q}_{103}$-GFP. Symbols in f: filled circles, $\mathrm{Htt}^{\mathrm{ex} 1}-\mathrm{Q}_{17}$-GFP; open circles, $\mathrm{Htt}^{\mathrm{ex} 1}-\mathrm{Q}_{47}-\mathrm{GFP}$; filled triangles, $\mathrm{Htt}^{\mathrm{ex} 1}-\mathrm{Q}_{103}-\mathrm{GFP}$. Asterisks in $\mathbf{c}-\mathbf{f}$ indicate $P<0.0001$. 
than double that for $\mathrm{Htt}^{\mathrm{ex} 1}-\mathrm{Q}_{47}-\mathrm{GFP}$ (Fig. 2e). Expansion from 47 to 103 glutamine residues had a larger effect on the incidence (Fig. 2e) than on the prevalence of IBs (Fig. 2d). Importantly, the polyQ-expansion-dependent risk of death was better correlated with the initiation of IB formation than with IB load (Figs 1d and 2e; Supplementary Fig. S3). This finding indicates that the principal toxic species might be an early IB intermediate or a form of diffuse intracellular Htt.

Is IB formation even necessary for polyQ-expansion-dependent death? IB formation has been dissociated from polyQ-dependent death $^{5,6,38,39}$, but the lack of longitudinal, single-cell analysis and the potential nonspecific effects of exogenous manipulations left the interpretation of these experiments in doubt ${ }^{17}$. For example, if IB formation accelerates death, neurons might die too rapidly to be detected. However, experiments in which we collected images every $2 \mathrm{~h}$ showed that only $1 \%$ of neurons that formed an IB within a $24-\mathrm{h}$ interval also died within that period. In fact, most neurons that form IBs can be followed for at least 2 days $\left(\mathrm{Htt}^{\mathrm{ex} 1}-\mathrm{Q}_{47}-\mathrm{GFP}\right.$, $71 \pm 4 \%$; $\mathrm{Htt}^{\mathrm{ex} 1}-\mathrm{Q}_{103}-\mathrm{GFP}, 55 \pm 4 \%$ ( \pm s.d.)). Thus, neurons that form IBs did not die too quickly for us to detect them. Moreover, survival analysis of Htt-transfected neurons that do not form IBs showed an increased risk of death among neurons transfected with $\mathrm{Htt}^{\mathrm{ex} 1}-\mathrm{Q}_{47}-\mathrm{GFP}$ or $\mathrm{Htt}^{\mathrm{ex} 1}-\mathrm{Q}_{103}-\mathrm{GFP}$ but not $\mathrm{Htt}^{\mathrm{ex} 1}-\mathrm{Q}_{17}-\mathrm{GFP}$ (Fig. 2f). These findings indicate that IB formation is not required for polyQ-expansion-dependent neuronal death and that other less aggregated or possibly monomeric species of polyQ-expanded Htt are toxic.

\section{Levels of diffuse Htt govern survival}

If the principal toxic species of Htt are distributed diffusely within neurons, their levels might be better predictors of neuronal death than IB formation. To determine whether GFP fluorescence can be used to quantify levels of GFP-tagged protein in single cells ${ }^{40}$, we performed three experiments. Both population-based and singlecell approaches showed that GFP fluorescence predicted the levels of GFP or of Htt to which it was attached (Fig. 3a, b, Supplementary Fig. S4). We conclude that we can quantify the amount of Htt protein within living neurons by imaging the fluorescence of the GFP tag.

To determine the relationship between levels of Htt and neuronal longevity, we used Cox proportional hazard analysis of neurons transfected with $\mathrm{Htt}^{\mathrm{ex1}}-\mathrm{Q}_{47}-\mathrm{GFP}$. The $\mathrm{Q}_{47}$ expansion is more typical among HD patients than $\mathrm{Q}_{72}$ or $\mathrm{Q}_{103}$. $\mathrm{Htt}^{\mathrm{ex1}}-\mathrm{Q}_{47}-\mathrm{GFP}$ also leads to death more slowly than the longer expansions, increasing our ability to resolve relationships between its expression and survival or IB formation. Cox proportional hazard analysis was used because it can determine whether and to what extent levels of Htt at an early time point within individual neurons can predict the longevities of those same neurons. We measured fluorescence from diffuse $\mathrm{Htt}$ within neurons, excluding IB fluorescence because Htt within IBs might have a different bioactivity. The levels of diffuse $\mathrm{Htt}^{\mathrm{ex} 1}-\mathrm{Q}_{47^{-}}$ GFP in neurons on the first day after transfection were correlated significantly and negatively with lifespan (Fig. 3c). The amounts of GFP alone (Fig. 3d) or $\mathrm{Htt}^{\mathrm{ex} 1}-\mathrm{Q}_{17}$-GFP (not shown) were not predictive. To exclude the possibility that neuron-subtype differences in vulnerability were required for the relationship we observed, we performed similar experiments in the homogeneous PC12 cell line. As in neurons, levels of $\mathrm{Htt}^{\text {ex1 }}-\mathrm{Q}_{47}-\mathrm{GFP}$ on the first day of survival analysis were a significant and negative predictor of survival, whereas the expression of the co-transfected marker protein, mRFP, had no predictive value (Fig. 3e). These results suggest that more diffuse forms of polyQ-expanded Htt are the principal toxic species and that their levels govern neuronal survival.

PolyQ expansions in ataxin-7 might cause toxicity by stabilizing ataxin-7, causing soluble forms to accumulate ${ }^{41}$. Could a similar effect explain how levels of diffuse polyQ-expanded Htt predict death? We measured the level of diffuse Htt in neurons before IBs had formed to avoid potential confounding effects of IB formation on these measurements. In contrast to findings with ataxin-7 (ref. 41), polyQ expansion was correlated with lower levels of diffuse $\mathrm{Htt}^{\text {ex1 }}$-GFP (Fig. 3f); similar results have been reported in HD (ref. 42). Thus, the effects of polyQ expansion on the levels of Htt do not explain polyQ-expansion-dependent neuronal death. Rather, they indicate that the polyQ expansion confers toxicity on more diffuse forms of $\mathrm{Htt}$ independently of its overall effect on the number of Htt molecules.

\section{IB formation prolongs survival}

Correlations between polyQ expansion and IB formation or neuronal death could suggest that IBs are pathogenic. Indeed, the levels of diffuse $\mathrm{Htt}^{\mathrm{ex} 1}-\mathrm{Q}_{47}-\mathrm{GFP}$ on day 1 after transfection were
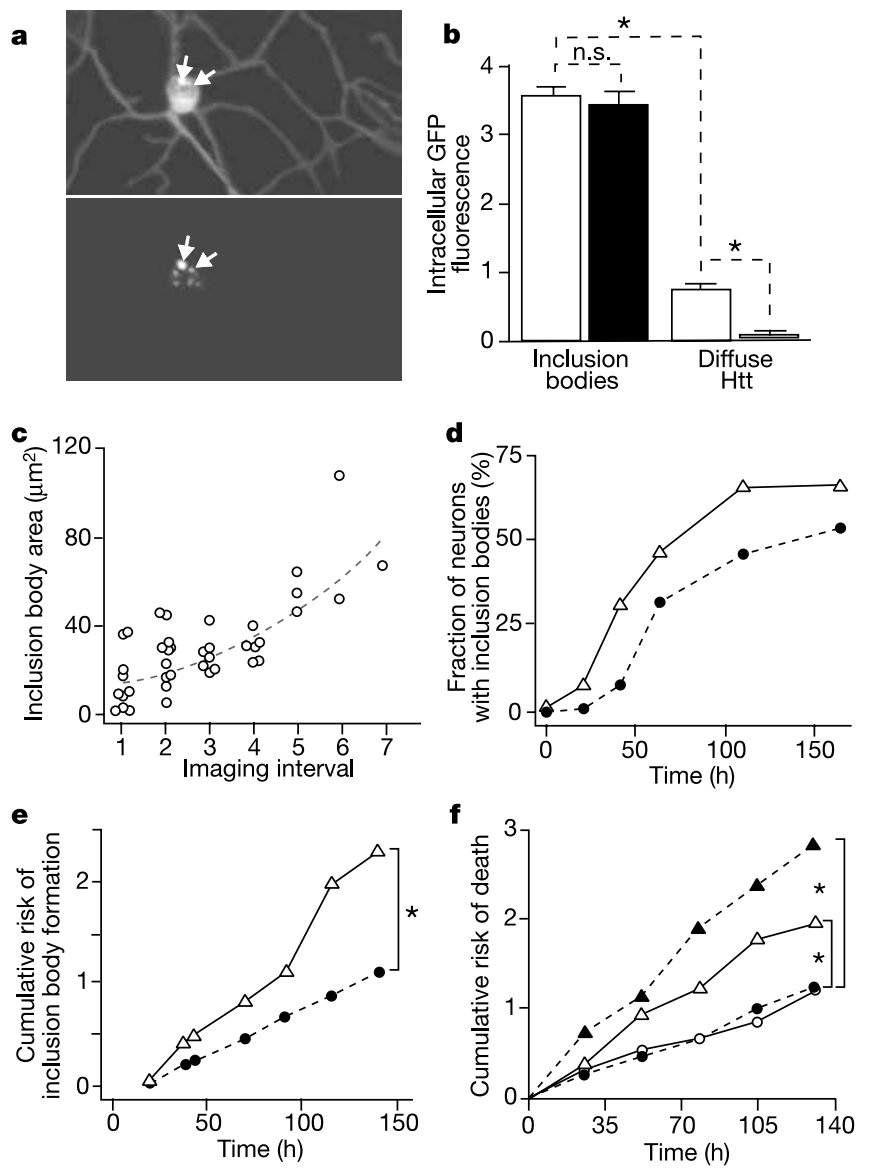

Figure 2 Many neurons die without forming IBs. a, Some neurons transfected with GFP-tagged versions of $\mathrm{Htt}$ with disease-associated polyQ expansions form highly fluorescent intracellular spheroid structures (arrows, top panel). Detergent treatment destroys GFP fluorescence except in these intracellular structures, indicating that they are IBs (arrows, bottom panel). b. Fluorescence intensity within IBs is very high, making it possible to monitor IBs in living neurons. A.u., arbitrary units of fluorescence intensity; n.s., not significant; $n=10-541$ neurons, three experiments. Open bars, in live neurons; filled bars, after detergent treatment. Error bars indicate s.e.m. c, IB growth was measured daily ( $n=12)$. d, A cohort of neurons was monitored longitudinally. The fraction of neurons with IBs grows with time and is greater for those transfected with $\mathrm{Htt}^{\mathrm{ex} 1}-\mathrm{Q}_{103}-\mathrm{GFP}$ (triangles) than $\mathrm{Htt}^{\mathrm{ex} 1}-\mathrm{Q}_{47}-\mathrm{GFP}$ (circles) (three experiments).

e, Cumulative risk of IB formation for $\mathrm{Htt}^{\mathrm{ex} 1}-\mathrm{Q}_{103}$-GFP (triangles) is about double that for $\mathrm{Htt}^{\mathrm{ex} 1}-\mathrm{Q}_{47}$-GFP (circles) and parallels the cumulative risk curves for survival ( $n=680$ neurons, three experiments). f, Neurons transfected with $\mathrm{Htt}$ that do not form detectable IBs nevertheless exhibit a significant polyQ-expansion-dependent increase in cumulative risk of death, indicating decreased survival ( $n=480$ neurons, three experiments). Filled triangles, $\mathrm{Htt}^{\mathrm{ex} 1}-\mathrm{Q}_{103}$-GFP; open triangles, $\mathrm{Htt}^{\mathrm{ex} 1}-\mathrm{Q}_{47}$-GFP; filled circles, $\mathrm{Htt}^{\mathrm{ex} 1}-\mathrm{Q}_{17}-\mathrm{GFP}$; open circles, GFP. Asterisks in $\mathbf{b}, \mathbf{e}$ and $\mathbf{f}$ represent $P<0.0001$. 
significantly and negatively correlated with the time of IB formation (Fig. 4a). Thus, levels of diffuse $\mathrm{Htt}^{\mathrm{ex1}}-\mathrm{Q}_{47}$-GFP predict whether and when an IB forms and also longevity. However, the same patterns might be expected if IB formation were a cellular response to cope with more diffuse, toxic forms of Htt. By analysing images of neurons as they formed IBs, we found that levels of diffuse Htt-GFP elsewhere in the cell fell rapidly after an IB appeared (Fig. 1a, bottom row). Within a day or two, diffuse Htt was nearly undetectable (Fig. 4b), and the rapid decrease in diffuse GFP fluorescence was directly correlated with the rapid growth of the IB (Fig. 1a, white arrow in bottom row, compare $85 \mathrm{~h}$ with $109 \mathrm{~h}$ ). In a few cases, several days after diffuse Htt was undetectable, the IB disappeared altogether (Fig. 1a, yellow arrow in bottom row).

For a direct investigation of the relationship between IB formation and the risk of death, we compared the survival curves of neurons that did or did not develop IBs. If IBs are pathogenic, neurons that develop them should die sooner than those that do
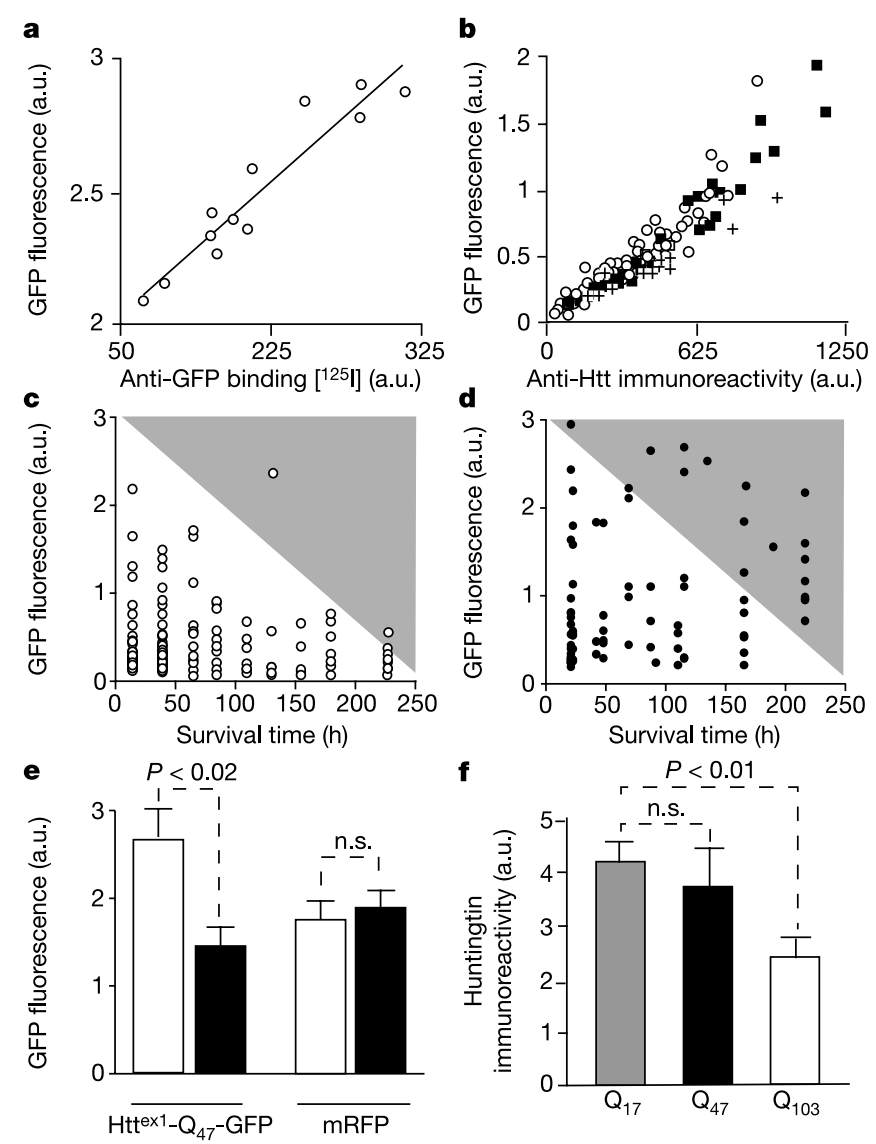

Figure $\mathbf{3}$ Levels of diffuse mutant Htt protein predict neuronal death. a, Cellular GFP fluorescence is well correlated with western blot measures of GFP within the same cells $(n=2): r^{2}=0.9 ; P<0.001$. b. Single-neuron levels of Htt fused to GFP estimated by imaging GFP fluorescence are well correlated with measurements by

immunocytochemistry $(n=2)$. Open circles, $\mathrm{Htt}^{\mathrm{ex} 1}-\mathrm{Q}_{17}$-GFP $\left(r^{2}=0.8\right)$; filled squares, $\mathrm{Htt}^{\mathrm{ex}}-\mathrm{Q}_{47}$-GFP $\left(r^{2}=0.9\right)$; crosses, $\mathrm{Htt}^{\mathrm{ex} 1}-\mathrm{Q}_{103}$-GFP $\left(r^{2}=0.9\right)$. c, Levels of diffuse $\mathrm{Htt}{ }^{\mathrm{ex}}-\mathrm{Q}_{47}$-GFP are a significant $(P<0.003)$ and negative predictor of neuronal longevity. Fluorescence of diffuse $\mathrm{Htt}^{\mathrm{ex} 1}-\mathrm{Q}_{47}$-GFP was measured in individual neurons ( $n=217$ neurons, three experiments) on the first day after transfection and plotted against their respective survival times. $\mathbf{d}$, Levels of GFP alone are not correlated with neuronal survival ( $n=97$ neurons, three experiments). e, Levels of $\mathrm{Htt}^{\mathrm{ex} 1}-\mathrm{Q}_{47}-\mathrm{GFP}$ but not the co-transfected marker, mRFP, are a significant and negative predictor of which PC12 cells live longer than $72 \mathrm{~h}(n=75)$. Open bars, less than $24 \mathrm{~h}$; filled bars, more than $72 \mathrm{~h}$. $\mathbf{f}$, Mean levels of $\mathrm{Htt}^{\mathrm{ex} 1}$-GFP are significantly and negatively correlated with the length of the polyQ stretch within $\mathrm{Htt}^{\mathrm{ex} 1}$ ( $n>90$ neurons, three experiments). Error bars in $\mathbf{e}$ and $\mathbf{f}$ indicate s.e.m. not. If IB formation is beneficial, the reverse might be true, and if IB formation is incidental, there might be no correlation with survival. To avoid selection bias, we identified all neurons that were alive at a particular time during the survival analysis and followed their fates prospectively. Neurons that contained or lacked an IB on the second day after transfection had similar risks of death (Fig. 4c).

However, on closer examination, we found that the subpopulation of neurons that form IBs on the second day also began with significantly higher intracellular levels of Htt-GFP (Fig. 4d). Thus, although the survival curves of the two populations were indistinguishable, the survival of neurons that formed IBs was better than that predicted by the relatively high initial expression of Htt-GFP (Fig. 3c). To test this idea further, we identified the a

c
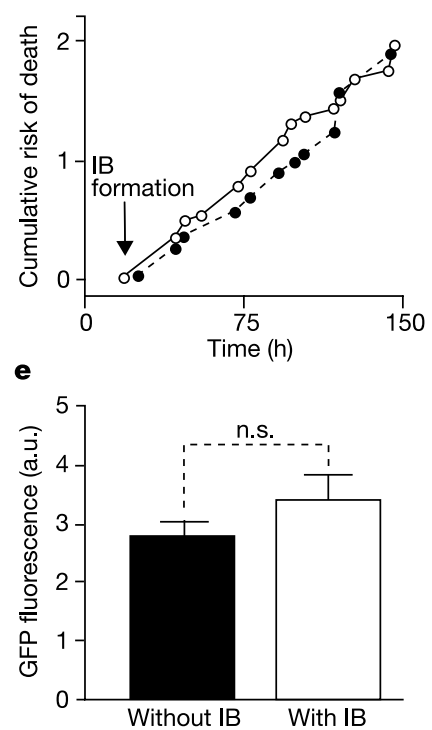

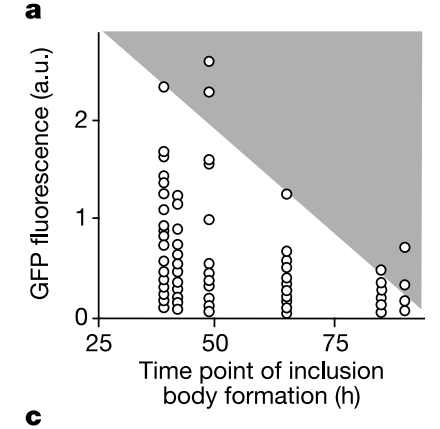

b

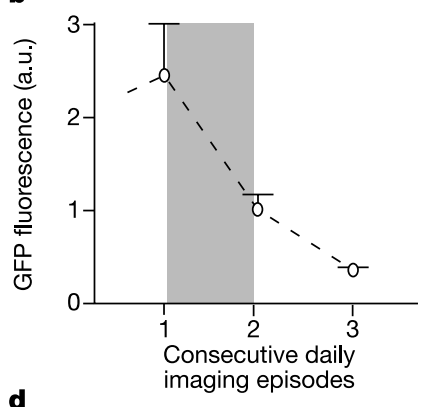

d

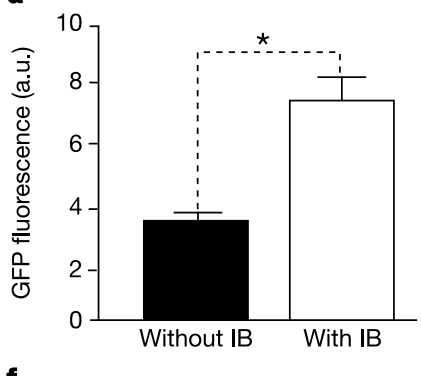

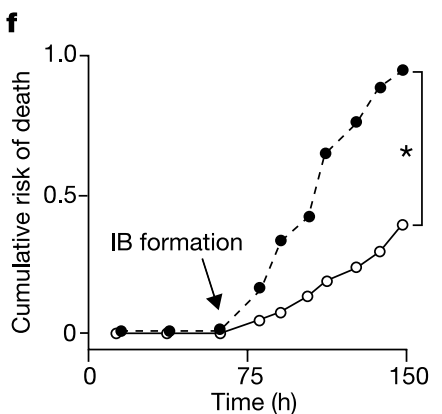

Figure $4 \mathrm{IB}$ formation is associated with decreased intracellular levels of diffuse $\mathrm{Htt}{ }^{\mathrm{ex}}$ and improved neuronal survival. a, Levels of diffuse $\mathrm{Htt}^{\mathrm{ex}}{ }^{\mathrm{a}} \mathrm{Q}_{47}$-GFP are correlated $(P<0.003)$ with IB formation ( $n=105$, three experiments). b. GFP fluorescence within single neurons was measured over a region adjacent to the site of IB formation. Upon IB formation (area shown in grey), levels of $\mathrm{Htt}^{\mathrm{ex} 1}-\mathrm{Q}_{47}$-GFP elsewhere in the neuron decreased rapidly $(n=10)$. c, Neurons transfected with $\mathrm{Htt}^{\mathrm{ex} 1}-\mathrm{Q}_{47}$-GFP were divided into two cohorts depending on whether they contained an IB (open circles) or not (filled circles) on the second day they were imaged. The risk of death and the overall survival of neurons in these two cohorts were not significantly different $(n=193$ neurons, three experiments). d, Neurons transfected with $\mathrm{Htt}^{\mathrm{ex}}-\mathrm{Q}_{47}$-GFP that contained an IB on the second day also began with significantly (asterisk, $P<0.001$ ) higher levels of $H \mathrm{Ht}^{\mathrm{e} \times 1}-\mathrm{Q}_{47}-\mathrm{GFP}$ than the cohort of neurons without an IB on the second day. e, Neurons transfected with $\mathrm{Htt}^{\mathrm{e} \times 1}-\mathrm{Q}_{47}$-GFP that formed IBs on the fourth day began with about the same levels of $\mathrm{Htt}^{\mathrm{ex} 1}-\mathrm{Q}_{47}$-GFP as the cohort of neurons that were alive on the fourth day but did not have IBs. $\mathbf{f}$, IB formation is associated with reduced death risk and increased survival among neurons transfected with $\mathrm{Htt}^{\mathrm{ex} 1}-\mathrm{Q}_{47}$-GFP that are alive beginning on the fourth day ( $n=224$ neurons, three experiments). Open circles, with an IB; filled circles, without an IB. Asterisk, $P<0$.0003. Error bars in $\mathbf{b}$, $\mathbf{d}$ and $\mathbf{e}$ indicate s.e.m. 
subpopulations of living neurons that either did or did not form an IB on the second day and that had similar initial levels of Htt-GFP. Prospective analysis revealed that neurons that formed IBs on the second day survived significantly longer than adjacent neurons that did not (Supplementary Fig. S5).

To further distinguish the contributions of Htt-GFP expression and IB formation to neuronal survival, we compared subpopulations of neurons with more closely matched levels of Htt-GFP. On either the fourth or sixth day after transfection, all living neurons started with similar levels of Htt-GFP, irrespective of whether they had developed an IB (Fig. 4e). We followed the survival of each of these populations prospectively. Neurons that formed an IB on either the fourth or sixth day survived significantly longer than adjacent neurons, which were otherwise similar but without an IB. IB formation was associated with a decrease in the cumulative risk of death (Fig. 4f, Supplementary Figs S6 and S7) to that seen with wild-type $\mathrm{Htt}\left(\mathrm{Htt}^{\mathrm{ex1}}-\mathrm{Q}_{17}\right.$-GFP, data not shown). Moreover, PC12 cells that formed IBs survived significantly longer than those that did not, indicating that neuron-subtype differences in IB formation and viability were not required for the relationship we observed (Supplementary Fig. S8). IB formation was generally associated with a decrease in more diffuse forms of intracellular $\mathrm{Htt}$ and a corresponding improvement in survival.

In our cellular model, IBs form in the cytoplasm and in the nucleus, as in HD. The nucleus seems to be an important site of toxicity for mutant $\mathrm{Htt}^{5,43,44}$. IBs could therefore be pathogenic in one location and beneficial in another. Analysis of neurons with cytoplasmic or nuclear IBs showed similar survival curves for both populations, and both survived significantly longer than neurons without IBs (data not shown). Thus, IB formation predicted increased survival regardless of the subcellular location.

\section{Discussion}

Using survival analysis, we found that neurons die from $\mathrm{Htt}$ protein in a manner best predicted by the level of diffuse forms of $\mathrm{Htt}$ and by the length of their polyQ expansions. PolyQ expansion increased the risk of death independently of its effect on the intracellular level of diffuse Htt. Surprisingly, IB formation reduced intracellular levels of diffuse Htt and prolonged survival. Together, these findings indicate that IB formation might protect neurons by decreasing the levels of toxic diffuse forms of mutant Htt (Fig. 5). The model is consistent with observations from post-mortem HD tissue, which reveal that IBs were more frequent in subpopulations of neurons that disproportionately survived ${ }^{38}$. It remains unclear whether levels fall because of autophagy ${ }^{45}$, because IBs sequester diffuse Htt or because IB formation is part of an adaptive programme that promotes increased Htt turnover ${ }^{8}$. Our results support the hypothesis that manipulations that improve survival and decrease IB formation (for example, certain aggregation inhibitors) might do so by interfering with the formation of toxic diffuse $\mathrm{Htt}$ species or with their ability to act on critical intracellular cellular targets ${ }^{46-48}$. Although our data do not exclude a non-cell-autonomous role for IBs in pathogenesis, the appearance of IBs in unrelated neurodegenerative diseases, such as Parkinson's disease and HD, might reflect a common coping response by neurons to diffuse toxic protein instead of a common pathogenic mechanism ${ }^{12}$.

In this study, survival analysis was essential for explaining the complex relationships between Htt expression, IB formation and

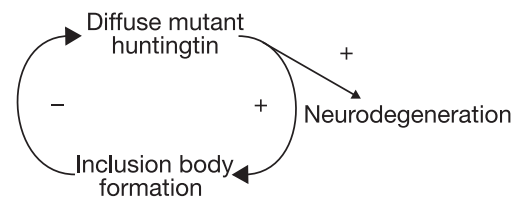

Figure $5 \mathrm{~A}$ model of the role of IB formation in huntingtin-induced neurodegeneration. neuronal death. This approach might prove particularly useful for disease-related research in which intermediate cellular and histological abnormalities can be clearly defined but whose precise relationship to pathogenesis can be obscure. Determining whether a particular change is pathogenic, incidental or beneficial has important implications for understanding mechanisms of disease and for identifying therapeutic targets. When pathogenesis is mediated by multiple effectors, survival analysis provides a way to quantify the contribution of each factor, potentially helping to assess its individual pathogenic significance.

\section{Methods}

\section{Plasmids}

Expression plasmids encoding an N-terminal fragment of Htt fused to GFP (pGW1$\mathrm{Htt}{ }^{\text {exl }}-\left[\mathrm{Q}_{25}, \mathrm{Q}_{47}, \mathrm{Q}_{72}\right.$ or $\left.\mathrm{Q}_{103}\right]-\mathrm{GFP}$ ) were derived from pcDNA3.1-based plasmids ${ }^{21}$ by subcloning into pGW1-CMV (British Biotechnologies). A PCR product of exon 1 of human Htt with 17 CAG repeats was ligated to GFP and used to create $\mathrm{pGW1}-\mathrm{Htt}^{\mathrm{exl}}-\mathrm{Q}_{17^{-}}$ GFP. A PCR product of $m R F P 1$ was ligated into pGW1-CMV to create pGW1-mRFP and into pcDNA3.1 $(+)$ to create $\mathrm{pcDNA3}$.1-mRFP. Plasmid constructions were confirmed by DNA sequencing.

\section{Cell culture and transfection}

Primary cultures of rat striatal neurons were prepared from embryos (embryonic days 16-18) and transfected with plasmids (6-7 days in vitro) as described ${ }^{5,49}$ (http:// gwebl.ucsf.edu/labs/finkbeiner). Typically, neurons were co-transfected with pGW1mRFP and a version of pGW1-Htt ${ }^{\mathrm{ex} 1}-\left[\mathrm{Q}_{17}, \mathrm{Q}_{25}, \mathrm{Q}_{47}, \mathrm{Q}_{72}\right.$ or $\left.\mathrm{Q}_{103}\right]-\mathrm{GFP}$ in a 1:1 molar ratio, using a total of $1-4 \mu \mathrm{g}$ of DNA in each well of a 24 -well plate. After transfection, neurons were maintained in serum-free medium.

To perform a modified LIVE-DEAD assay (Molecular Probes), growth medium was replaced with Eagle's basal medium $48 \mathrm{~h}$ after transfection. At $20 \mathrm{~min}$ before treatment with kainate (Sigma), ethidium homodimer ( $5 \mu \mathrm{M}$; Molecular Probes) was added, and images of transfected neurons were collected before and every $30 \mathrm{~min}$ after kainate addition. A detergent-resistance assay was performed as described ${ }^{21}$, with minor modifications. Neurons with putative IBs were imaged, treated with $1 \%$ paraformaldehyde for $15 \mathrm{~min}$ at $37^{\circ} \mathrm{C}$ followed by $5 \%$ Triton X-100 and $5 \%$ SDS for $20 \mathrm{~min}$ at $37^{\circ} \mathrm{C}$, and imaged again. PC12 cells inducibly expressing $\mathrm{Htt}^{\mathrm{ex}}-\mathrm{Q}_{25}$-GFP or $\mathrm{Htt}^{\mathrm{ex1}}-\mathrm{Q}_{103}-\mathrm{GFP}^{50}$ were plated at $10^{4}$ cells per $\mathrm{cm}^{2}$, transiently transfected with pcDNA3.1mRFP and induced with $1 \mu \mathrm{M}$ tebufenozide. In some experiments, wild-type PC12 cells were plated at $5 \times 10^{4}$ cells per $\mathrm{cm}^{2}$ and co-transfected with a version of pGW1-Htt ${ }^{\text {exl }}$. $\left[\mathrm{Q}_{17}, \mathrm{Q}_{25}, \mathrm{Q}_{47}, \mathrm{Q}_{72}\right.$ or $\left.\mathrm{Q}_{103}\right]$-GFP and pcDNA3.1-mRFP in a 1:1 molar ratio, using a total of $2 \mu \mathrm{g}$ of DNA in each well of a 24 -well plate.

\section{Immunocytochemistry}

Striatal neurons grown on 12-mm glass coverslips were examined $36 \mathrm{~h}$ after transfection as described $^{5}$, with anti-GFP (1:500 dilution; Chemicon), anti-Htt EM48 (1:50 dilution; Chemicon) and anti-chicken or anti-rabbit Cy3-labelled antibodies (1:300 dilution; Jackson Immunochemical).

\section{Western blots}

HEK-293 cells grown in DMEM medium containing 10\% calf serum, $2 \mathrm{mM}$ glutamine and penicillin/streptomycin $\left(100 \mathrm{U} \mathrm{ml}^{-1} / 100 \mu \mathrm{g} \mathrm{ml}^{-1}\right)$ were transiently transfected with pGW1-GFP (1-6 $\mu$ g per well). Images were captured every $24 \mathrm{~h}$ for 3 days. Protein extracts were prepared from cells immediately after imaging, subjected to SDS-polyacrylamide-gel electrophoresis, blotted with anti-GFP antibody (1:1000 dilution; Zymed) and detected with ${ }^{125}$ I-labelled secondary antibody and a PhosphoImager screen (Fuji).

\section{Robotic microscope imaging system}

The system is based on an inverted Nikon microscope (TE300 Quantum). Olympus $4 \times$ (numerical aperture 0.13 ) and $10 \times$ (numerical aperture 0.30 ) and Nikon $20 \times$ (numerical aperture 0.45$)$ objectives were used. Xenon lamp (175 W) illumination was supplied by a liquid light guide to reduce electrical noise. Images were detected and digitized with a Hamamatsu Orca II 12/14-bit, digital, cooled charge-coupled device camera and Universal Imaging Metamorph software. Stage movements and focusing were executed with computer-controlled stepper motors. Fluorescence excitation and emission filters were moved into or out of the optical path with each program loop by two tenposition filter wheels (Sutter Instruments) under computer control. The whole system is mounted on a vibration isolation table to reduce noise. Computer commands that perform and coordinate automated stage movements, filter wheel movements and focusing were generated with software programs that combine custom-designed and commercially available algorithms. Additional programs for image analysis were written with MatLab and Visual C software.

\section{Image and statistical analysis}

Measurements of Htt expression, IB formation and neuron survival were extracted from files generated with automated imaging by automated analysis programs or by visual inspection. Automated programs identified living transfected neurons by physical dimensions and fluorescence. IBs were monitored by size and fluorescence intensity. The expression of GFP-tagged versions of Htt was estimated by measuring GFP fluorescence intensity over a region of interest that corresponded to the cell soma or as otherwise 
indicated, using the fluorescence of co-transfected mRFP as a guide. These GFP intensity values were background-subtracted by using an adjacent area of the image.

For statistical analysis, survival time was defined as the imaging time point at which cell was last seen alive. Kaplan-Meier curves were used to estimate survival and hazard functions with commercially available software (Statview). Differences in Kaplan-Meier curves were assessed with the log-rank test. Linear regression was used to correlate $\mathrm{Htt}$ expression measured with different methods, and correlations between $\mathrm{Htt}$ expression and survival or IB formation were made with Cox proportional hazard analysis. Differences in mean measurements were compared by analysis of variance or $t$-test.

Received 9 July; accepted 6 September 2004; doi:10.1038/nature02998.

1. Davies, S. W. et al. Formation of neuronal intranuclear inclusions underlies the neurological dysfunction in mice transgenic for the HD mutation. Cell 90, 537-548 (1997).

2. Becher, M. W. et al. Intranuclear neuronal inclusions in Huntington's disease and dentatorubral and pallidoluysian atrophy_correlation between the density of inclusions and IT-15 CAG triplet repeat length. Neurobiol. Dis. 4, 387-397 (1998).

3. DiFiglia, M. et al. Aggregation of huntingtin in neuronal intranuclear inclusions and dystrophic neurites in brain. Science 277, 1990-1993 (1997).

4. Ordway, J. M. et al. Ectopically expressed CAG repeats cause intranuclear inclusions and a progressive late onset neurological phenotype in the mouse. Cell 91, 753-763 (1997).

5. Saudou, F., Finkbeiner, S., Devys, D. \& Greenberg, M. E. Huntingtin acts in the nucleus to induce apoptosis, but death does not correlate with the formation of intranuclear inclusions. Cell 95, 55-66 (1998).

6. Klement, I. A. et al. Ataxin-1 nuclear localization and aggregation: Role in polyglutamine-induced disease in SCA1 transgenic mice. Cell 95, 41-53 (1998).

7. Cummings, C. J. et al. Mutation of the E6-AP ubiquitin ligase reduces nuclear inclusion frequency while accelerating polyglutamine-induced pathology in SCA1 mice. Neuron 24, 879-892 (1999).

8. Taylor, J. P. et al. Aggresomes protect cells by enhancing the degradation of toxic polyglutaminecontaining protein. Hum. Mol. Genet. 12, 749-757 (2003).

9. Shimohata, T. et al. Expanded polyglutamine stretches form an 'aggresome'. Neurosci. Lett. 323, 215-218 (2002).

10. Sisodia, S. S. Nuclear inclusions in glutamine repeat disorders: Are they pernicious, coincidental or beneficial? Cell 95, 1-4 (1998).

11. Bence, N. F., Sampat, R. M. \& Kopito, R. R. Impairment of the ubiquitin-proteasome system by protein aggregation. Science 292, 1552-1555 (2001).

12. Ross, C. A. Intranuclear neuronal inclusions: A common pathogenic mechanism for glutamine-repeat neurodegenerative diseases. Neuron 19, 1147-1150 (1997).

13. Poirier, M. A. et al. Huntingtin spheroids and protofibrils as precursors in polyglutamine fibrilization. J. Biol. Chem. 277, 41032-41037 (2002).

14. Chen, S., Berthelier, V., Yang, W. \& Wetzel, R. Polyglutamine aggregation behavior in vitro supports a recruitment mechanism of cytotoxicity. J. Mol. Biol. 311, 173-182 (2001).

15. Wyttenbach, A. et al. Effects of heat shock, heat shock protein 40 (HDJ-2), and proteasome inhibition on protein aggregation in cellular models of Huntington's disease. Proc. Natl Acad. Sci. USA 97, $2898-2903$ (2000)

16. Muchowski, P. J., Ning, K., D'Souza-Schorey, C. \& Fields, S. Requirement of an intact microtubule cytoskeleton for aggregation and inclusion body formation by a mutant huntingtin fragment. Proc Natl Acad. Sci. USA 99, 727-732 (2002).

17. Perutz, M. F. \& Windle, A. H. Cause of neural death in neurodegenerative disease attributable to expansion of glutamine repeats. Nature 412, 143-144 (2001).

18. Arrasate, M., Brooks, L., Chang, P., Mitra, S. \& Finkbeiner, S. Longitudinal analysis to identify pathogenic factors in a striatal model of Huntington's disease. Soc. Neurosci. Abstr. 29, 209.8 (2003). 19. Collett, D. Modeling Survival Data in Medical Research (Chapman \& Hall, London, 1994).

20. Therneau, T. M. \& Grambsch, P. M. Modeling Survival Data: Extending the Cox Model (Springer, New York, 2000).

21. Kazantsev, A., Preisinger, E., Dranovsky, A., Goldgaber, D. \& Housman, D. Insoluble detergentresistant aggregates form between pathological and nonpathological lengths of polyglutamine in mammalian cells. Proc. Natl Acad. Sci. USA 96, 11404-11409 (1999).

22. Goldberg, Y. P. et al. Cleavage of huntingtin by apopain, a proapoptotic cysteine protease, is modulated by the polyglutamine tract. Nature Genet. 13, 442-449 (1996).

23. Scherzinger, E. et al. Huntingtin-encoded polyglutamine expansions form amyloid-like protein aggregates in vitro and in vivo. Cell 90, 549-558 (1997).

24. Wellington, C. L. et al. Caspase cleavage of gene products associated with triplet expansion disorder generates truncated fragments containing the polyglutamine. J. Biol. Chem. 273, 9158-9167 (1998).

25. Kim, Y. J. et al. Caspase 3-cleaved N-terminal fragments of wild-type and mutant huntingtin are present in normal and Huntington's disease brains, associate with membranes, and undergo calpaindependent proteolysis. Proc. Natl Acad. Sci. USA 98, 12784-12789 (2001).

26. Mende-Mueller, L. M., Toneff, T., Hwang, S. R., Chesselet, M. F. \& Hook, V. Y. H. Tissue-specific proteolysis of huntingtin (htt) in human brain: Evidence of enhanced levels of $\mathrm{N}$ - and $\mathrm{C}$-terminal htt fragments in Huntington's disease striatum. J. Neurosci. 21, 1830-1837 (2001).

27. Mangiarini, L. et al. Exon 1 of the HD gene with an expanded CAG repeat is sufficient to cause a progressive neurological phenotype in transgenic mice. Cell 87, 493-506 (1996).
8. Campbell, R. E. et al. A monomeric red fluorescent protein. Proc. Natl Acad. Sci. USA 99, 7877-7882 (2002)

29. Strebel, A., Harr, T., Bachmann, F., Wernli, M. \& Erb, P. Green fluorescent protein as a novel tool to measure apoptosis and necrosis. Cytometry 43, 126-133 (2001).

30. MacDonald, M. E. in Trinucleotide Diseases and Instability (ed. Oostra, B. A.) 47-75 (Springer, Berlin, 1998).

31. Clarke, G. et al. A one-hit model of cell death in inherited neuronal degenerations. Nature 406, 195-199 (2000).

32. Reiner, A. et al. Differential loss of striatal projection neurons in Huntington disease. Proc. Natl Acad. Sci. USA 85, 5733-5737 (1988).

33. Richfield, E. K., Maguire-Zeiss, K. A., Vonkeman, H. E. \& Voorn, P. Preferential loss of preproenkephalin versus preprotachykinin neurons from the striatum of Huntington's disease patients. Ann. Neurol. 38, 852-861 (1995).

34. Rajan, R. S., Illing, M. E., Bence, N. F. \& Kopito, R. R. Specificity in intracellular protein aggregation and inclusion body formation. Proc. Natl Acad. Sci. USA 98, 13060-13065 (2001).

35. Moulder, K. L., Onodera, O., Burke, J. R., Strittmatter, W. J. \& Johnson, E. M. Jr Generation of neuronal intranuclear inclusions by polyglutamine-GFP: Analysis of inclusion clearance and toxicity as a function of polyglutamine length. J. Neurosci. 19, 705-715 (1999).

36. Gutekunst, C. A. et al. Nuclear and neuropil aggregates in Huntington's disease: Relationship to neuropathology. J. Neurosci. 19, 2522-2534 (1999).

37. Preisinger, E., Jordan, B. M., Kazantsev, A. \& Housman, D. Evidence for a recruitment and sequestration mechanism in Huntington's disease. Phil. Trans. R. Soc. Lond. B 354, 1029-1034 (1999).

38. Kuemmerle, S. et al. Huntingtin aggregates may not predict neuronal death in Huntington's disease. Ann. Neurol. 46, 842-849 (1999).

39. Kim, M. et al. Mutant huntingtin expression in clonal striatal cells: Dissociation of inclusion formation and neuronal survival by caspase inhibition. J. Neurosci. 19, 964-973 (1999).

40. Hack, N. J. et al. Green fluorescent protein as a quantitative tool. J. Neurosci. Methods $95,177-184$ (2000).

41. Yoo, S.-Y. et al. SCA7 knockin mice model human SCA7 and reveal gradual accumulation of mutant ataxin-7 in neurons and abnormalities in short-term plasticity. Neuron 37, 383-401 (2003).

42. Persichetti, F. et al. Differential expression of normal and mutant Huntington's disease gene alleles. Neurobiol. Dis. 3, 183-190 (1996).

43. Kegel, K. B. et al. Huntingtin is present in the nucleus, interacts with the transcriptional corepressor C-terminal binding protein, and represses transcription. J. Biol. Chem. 277, 7466-7476 (2002).

44. Peters, M. F. et al. Nuclear targeting of mutant huntingtin increases toxicity. Mol. Cell. Neurosci. 14, 121-128 (1999).

45. Ravikumar, B. et al. Inhibition of mTOR induces autophagy and reduces toxicity of polyglutamine expansions in fly and mouse models of Huntington disease. Nature Genet. 36, 585-595 (2004)

46. Sánchez, I., Mahlke, C. \& Yuan, J. Pivotal role of oligomerization in expanded polyglutamine neurodegenerative disorders. Nature 421, 373-379 (2003).

47. Apostol, B. L. et al. A cell-based assay for aggregation inhibitors as therapeutics of polyglutaminerepeat disease and validation in Drosophila. Proc. Natl Acad. Sci. USA 100, 5950-5955 (2003).

48. Tanaka, M. et al. Trehalose alleviates polyglutamine-mediated pathology in a mouse model of Huntington disease. Nature Med. 10, 148-154 (2004).

49. Finkbeiner, S. et al. CREB: A major mediator of neuronal neurotrophin responses. Neuron 19, 1031-1047 (1997).

50. Aiken, C. T., Tobin, A. J. \& Schweitzer, E. S. A cell-based screen for drugs to treat Huntington's disease. Neurobiol. Dis. 16, 546-555 (2004).

Supplementary Information accompanies the paper on www.nature.com/nature.

Acknowledgements We thank A. Kazantzev, D. Housman and the Hereditary Disease Foundation for pcDNA3.1-Htt $\left(\mathrm{Q}_{25}, \mathrm{Q}_{47}, \mathrm{Q}_{72}, \mathrm{Q}_{103}\right)$-GFP plasmids; R. Truant for the PCR template (GFP-109-17Q- $\beta$ gal) used to create $\mathrm{pGW} 1-\mathrm{Htt}^{\mathrm{exl}}-\mathrm{Q}_{17}-\mathrm{GFP}$; R. Tsien for $m R F P$ cDNA D. Bredesen, S. Prusiner, S. Lindquist, R. Edwards, A. Tobin, E. Signer, C. Johnson, P. Muchowski and members of the Finkbeiner laboratory for useful discussions; S. Ordway and G. Howard for editorial assistance; K. Nelson for administrative assistance; and E. Oliver and D. Murphy for their interest and support. Primary support for this work was provided by the National Institute of Neurological Disease and Stroke (S.F). Additional support was provided by the National Institute of Aging and the J. David Gladstone Institutes (S.F.). M.A. is a MECD-Fulbright Fellow and is supported by the Hillblom Foundation. S.M. is supported by the NIH-NIGMS UCSF Medical Scientist Training Program and a fellowship from the UCSF Hillblom Center for the Biology of Aging. E.S. is supported by the National Institute of Neurological Disease and Stroke, the Hereditary Disease Foundation, and the High Q Foundation.

Competing interests statement The authors declare that they have no competing financial interests.

Correspondence and requests for materials should be addressed to S.F.

(sfinkbeiner@gladstone.ucsf.edu) 